\title{
Divergent creative thinking in young and older adults: Extending the effects of an episodic specificity induction
}

\author{
Kevin P. Madore ${ }^{1,2}$ (I) $\cdot$ Helen G. Jing ${ }^{1} \cdot$ Daniel L. Schacter ${ }^{1}$
}

Published online: 21 March 2016

(C) Psychonomic Society, Inc. 2016

\begin{abstract}
Recent research has suggested that an episodic specificity induction - brief training in recollecting the details of a past experience - enhances divergent creative thinking on the alternate uses task (AUT) in young adults, without affecting performance on tasks thought to involve little divergent thinking; however, the generalizability of these results to other populations and tasks is unknown. In the present experiments, we examined whether the effects of an episodic specificity induction would extend to older adults and a different index of divergent thinking, the consequences task. In Experiment 1, the specificity induction significantly enhanced divergent thinking on the AUT in both young and older adults, as compared with a control induction not requiring specific episodic retrieval; performance on a task involving little divergent thinking (generating associates for common objects) did not vary as a function of induction. No overall age-related differences were observed on either task. In Experiment 2, the specificity induction significantly enhanced divergent thinking (in terms of generating consequences of novel scenarios) in young adults, relative to another control induction not requiring episodic retrieval. To examine the types of creative ideas affected by the induction, the participants in both experiments
\end{abstract}

Electronic supplementary material The online version of this article (doi:10.3758/s13421-016-0605-z) contains supplementary material, which is available to authorized users.

Kevin P. Madore

madore@fas.harvard.edu

1 Department of Psychology and Center for Brain Science, Harvard University, Cambridge, MA, USA

2 Department of Psychology, Harvard University, 33 Kirkland Street, Cambridge, MA 02138, USA also labeled each of their divergent-thinking responses as an "old idea" from memory or a "new idea" from imagination. New, and to some extent old, ideas were significantly boosted following the specificity induction relative to the control. These experiments provide novel evidence that an episodic specificity induction can boost divergent thinking in young and older adults, and indicate that episodic memory is involved in multiple divergent-thinking tasks.

Keywords Episodic specificity induction $\cdot$ Aging $\cdot$ Episodic memory $\cdot$ Creativity $\cdot$ Divergent thinking

Divergent thinking refers to the ability to generate creative ideas by combining diverse types of information in novel ways (Guilford, 1967). Studies of divergent thinking have covered a wide range of topics, including the relation of divergent thinking to personality characteristics (e.g., McCrae, 1987), the properties of tests for assessing divergent thinking (e.g., Runco, Okuda, \& Thurston, 2014), the effects of task instructions on creative responding (e.g., Nusbaum, Silvia, \& Beaty, 2014), and the neural correlates of divergent thinking (e.g., Benedek et al., 2014).

During the past few years, a growing number of studies have begun to examine how divergent thinking is influenced by episodic memory, classically characterized by Tulving $(1983,2002)$ as a neurocognitive system that supports the recollection of specific experiences. Several kinds of evidence suggest a link between divergent thinking and episodic memory: When performing the alternate uses task (AUT), a frequently used measure of divergent thinking (Guilford, 1967), participants sometimes report episodic memories (Gilhooly, Fioratou, Anthony, \& Wynn, 2007; Runco \& Acar, 2010); the number of uses generated on the AUT is positively correlated with the number of episodic details that participants 
report when they imagine future experiences (Addis, Pan, Musicaro, \& Schacter, 2016); and amnesic patients with episodic memory deficits exhibit impaired divergent thinking on the Torrance Tests of Creative Thinking (Duff, Kurczek, Rubin, Cohen, \& Tranel, 2013).

Building on these observations, Madore, Addis, and Schacter (2015) recently provided evidence for a link between episodic memory and divergent thinking using a new approach that has been referred to as an episodic specificity induction: brief training in recollecting the episodic details of previous experiences (i.e., who, what, where, and when details). The specificity induction is based on the cognitive interview (CI; Fisher \& Geiselman, 1992), a protocol used mainly in forensic contexts to increase episodic retrieval in eyewitnesses. When receiving this induction, participants are encouraged to focus on episodic details pertaining to people, objects, and actions in a recently viewed video of an everyday scene (i.e., people performing actions in a kitchen setting), and subsequently perform seemingly unrelated cognitive tasks. The effect of the specificity induction on these tasks is compared with that of a control induction, which either requires participants to provide their general impressions of a recently viewed video or to solve math problems, neither of which involves detailed episodic retrieval. Several studies have shown that compared with these control inductions, the specificity induction selectively boosts the number of episodic details that participants provide on subsequent tasks that are thought to rely on episodic memory, including remembering past experiences, imagining future experiences, and solving personal problems, while having no effect on the number of semantic details (i.e., factual information not specific to time and place) that participants provide on these tasks, and no effect on tasks that are not thought to rely on episodic memory (Jing, Madore, \& Schacter, 2016; Madore, Gaesser, \& Schacter, 2014; Madore \& Schacter, 2014, 2016; for a review, see Schacter \& Madore, in press).

Applying the specificity induction to divergent thinking, Madore et al. (2015) administered specificity and control inductions prior to performance of the AUT and an object association task (OAT; based on Abraham et al., 2012) that requires participants to generate common associates of objects but requires little divergent thinking. In an initial experiment, the specificity induction produced a significant increase in the number of uses (i.e., alternative object uses that are possible and feasible) that participants generated on the AUT, while having no effect on performance on the OAT. A second experiment replicated this effect of the specificity induction on the AUT, while revealing no effect of the induction on a standard test of convergent thinking, the remote associates test (Mednick, 1962), which requires one semantic solution word to a given problem.

These results clearly support the idea that episodic memory influences divergent thinking. They are, however, somewhat limited for at least two reasons. First, both of the experiments reported by Madore et al. (2015) included only young adults. It would be desirable to know whether the effect of the specificity induction on divergent thinking extends to other participant populations. In Experiment 1, we assessed this issue by comparing, for the first time, the performance of a group of young adults with that of healthy older adults on a divergent-thinking task after participants had received specificity and control inductions. As we review below, considerable research has focused on divergent thinking in older adults, but little evidence is available concerning the contribution of episodic retrieval to divergent creative thinking in older adults. However, previous studies using the specificity induction have shown that older adults benefit as much from the induction as do young adults on tasks that tap memory, imagination, and problem solving (Madore et al., 2014; Madore \& Schacter, 2014). On the basis of this earlier work, we hypothesized that these benefits would extend to divergent thinking as assessed by the AUT. Second, previous specificity induction experiments and Experiment 1 in the present study all relied on the AUT to assess divergent thinking. It is important to determine whether the observed effects of the specificity induction are restricted to the AUT, or whether they extend to other divergent-thinking tasks. In Experiment 2, we assessed this issue by examining the effects of the specificity induction on the consequences task (Guilford, 1967; Torrance, 1962) for the first time. This task also allows us to characterize more precisely the kinds of ideas that are impacted by the specificity induction. Overall, examining the effects of the induction in terms of age (Exp. 1) and of a second creativity index (Exp. 2) should allow us to assess with greater certainty the strength of the evidence for a contribution of episodic retrieval to divergent thinking.

\section{Experiment 1}

The impact of age on divergent-thinking performance has been studied extensively, with mixed results. Some studies have suggested that older adults exhibit reduced performance relative to young adults on divergent-thinking tasks, particularly with respect to verbal generative outputs such as total ideas and ideas that are binned into unique categories (e.g., Alpaugh \& Birren, 1977; Alpaugh, Parham, Cole, \& Birren, 1982; McCrae, Arenberg, \& Costa, 1987; Palmiero, 2015; Reese et al., 2001; Ruth \& Birren, 1985); other studies have suggested that age group does not affect generative divergent thinking (e.g., Addis et al., 2016; Foos \& Boone, 2008; Leon et al., 2014; Palmiero, Di Giacomo, \& Passafiume, 2014; Roskos-Ewoldsen, Black, \& McCown, 2008). However, our focus was not so much on whether older adults exhibit intact or impaired divergent thinking overall, but rather on whether divergent-thinking performance in older adults, as assessed by the AUT, benefits from an episodic specificity induction to the same extent as that of young adults. 
This issue is important because, as far as we know, only one previous study has provided evidence regarding the relation between episodic retrieval and divergent thinking in older adults. Addis et al. (2016) reported that performance on the AUT in both young and older adults is associated with the amount of episodic detail in future imagined events, but not in past imagined events or past recalled events. We think that the episodic specificity induction that we have developed in previous studies is wellsuited to allow further exploration of the possible influences of episodic retrieval on divergent thinking in older adults, because the induction has been shown to selectively boost the numbers of episodic details that both young and older adults provide on tasks that require remembering past experiences, imagining future experiences, and solving personal problems, while having no effect on the generation of semantic details (Madore et al., 2014; Madore \& Schacter, 2014). Because these earlier studies showed that older adults benefit as much from a specificity induction as do young adults, we hypothesized that in the present experiment, after receiving the specificity induction relative to a control induction, participants from both age groups would show comparable boosts in measures of generative AUT performance that had shown induction effects previously in our study of young adults (Madore et al., 2015), including the total number of uses generated, appropriate uses (i.e., total number of uses that are feasible), categories of uses (i.e., total number of unique categories that uses can be binned under for each cue), and categories of appropriate uses (i.e., appropriate uses that are clustered into categories). By contrast, in our previous study we found no effects of the specificity induction on ratings of elaboration (i.e., a rating of how detailed each generated use is) or creativity (i.e., a rating of how original/unusual each generated use is), and thus we did not expect to find induction effects in either young or older adults on these measures.

To gain insight into the types of ideas generated by young and older adults on the AUT, we also had participants label each use they generated as either an "old" idea from memory (i.e., a novel use of an object that a participant had previously experienced or known about) or a "new" idea from imagination (i.e., a novel use that the participant had generated for the first time during the experiment). This procedure has been used in previous studies of the AUT by Benedek et al. (2014), Gilhooly et al. (2007), and Silvia, Nusbaum, and Beaty (2015); a similar approach was also taken by Runco and Acar (2010). Our previous study of young adults (Madore et al., 2015) revealed some evidence that the specificity induction boosts both types of ideas, but the effects were more consistent across two experiments for old than for new ideas. These findings thus suggest that the episodic processes targeted by the specificity induction may contribute both to retrieving use-related episodic memories and to constructing or imagining novel uses that have not been previously experienced, but more data are needed to evaluate this characterization. This idea fits within the framework of the constructive episodic simulation hypothesis (Schacter \&
Addis, 2007), which holds that elements of prior experiences may be drawn on and recombined in order to perform tasks that go beyond strict remembering, and that involve using the currency of past experiences in new ways. No previous studies have compared old and new idea generation in older adults, so we also sought to determine whether similar effects would be observed in both age groups. Because older adults have more past experiences to draw on than young adults do (see Leon et al., 2014, for a discussion), it is plausible that older adults might provide relatively more "old" than "new" ideas than would young adults.

\section{Method}

\section{Participants}

Twenty-four young adults (age $=18-30$ years, $M=21.61, S D$ $=2.95 ; 15$ female, nine male) and 24 older adults (age $=64$ 87 years, $M=71.83, S D=5.82 ; 17$ female, seven male) were recruited from the Boston area for Experiment 1. The young adults were recruited from a community research study pool (including students at Boston University and Harvard University), and the older adults were recruited from newspaper advertisements and community centers. All of the participants had normal or corrected-to-normal vision and no history of psychiatric or neurological impairment at the time of the study, and none had participated in a previous inductionrelated experiment. The older adults were also screened with an extensive neuropsychological battery prior to participating in the study and were considered cognitively healthy (MiniMental Status Examination scores of 25-30, $M=29.17, S D=$ 1.30). Educational levels were similar and did not significantly differ between the young adults $(M=15.35, S D=1.47)$ and the older adults $(M=15.65, S D=2.17)$. All participants provided written consent before completing the study and were treated in a manner approved by Harvard University's ethics board. Participants were paid or received course credit for study completion.

Prior to running the study, we ran a power analysis (G*Power 3; see Faul, Erdfelder, Lang, \& Buchner, 2007) to determine that 24 young adults and 24 older adults would be sufficient for observing a medium-sized induction-related effect within each age group ( $d=0.60$, power $>.80$, two-tailed $)$, and a large-sized, overall age-related effect between groups $(d$ $=0.80$, power $>.80$, one-tailed). This sample size is in line with previous induction-related aging work (Madore et al., 2014; Madore \& Schacter, 2014), in which induction-related effects within age groups and overall age-related effects between groups had been observed. At the end of the study, the data from one young adult and one older adult were excluded for task noncompliance and a technical glitch, respectively. Thus, 23 young adults and 23 older adults were included in the final analyses. 


\section{Overview}

Participants came to the lab for two sessions spaced at least a week apart $(M=7.71$ days, $S D=1.95$, median and mode $=7)$. In each session, participants (a) watched one of two short videos involving a man and a woman performing different activities in a kitchen, (b) received either the episodic specificity induction or the math control induction after watching the video, and (c) then completed the alternate uses (AUT), object association (OAT), and imagination main tasks. The video-induction order was counterbalanced across participants, and participants viewed different stimuli in each session. At the end of the second session, participants also labeled each alternative use they had generated as an "old idea" from memory or a "new idea" from imagination. Participants finished the study by completing category fluency and letter fluency measures and four questionnaires related to individual differences in creativity, personality, and memory (see the supplemental materials). Each session took on average 1.5 to $2 \mathrm{~h}$ to complete. Figure 1 illustrates the experimental sequence and variables of interest.

\section{Materials and procedure}

\section{Inductions}

Episodic specificity induction Half of the young adults and half of the older adults were randomly assigned to receive the episodic specificity induction in the first session after watching the video (and the control induction in the second session). The specificity induction included probes and instructions from the Cognitive Interview, a forensic protocol that has been used with young and older adult eyewitnesses to enhance accurate detail for remembered events (Fisher \& Geiselman, 1992; Memon et al., 2010). During the induction, participants were first told that they were the chief expert regarding the contents of the video they had watched. Then they were guided through three mental imagery probes in which they were asked to close their eyes, get a good mental picture in their mind, and report everything they could remember in as much detail as possible related to the setting, people, and actions of the video, respectively. For each probe category, participants were also asked open-ended, follow-up questions about elements of the video they had retrieved (see the supplemental materials for the full script).

Control induction The other half of the young and older adults were randomly assigned to receive the math control induction in the first session after watching the video (and the specificity induction in the second session). For this induction, participants completed addition and subtraction math problems and were not asked any questions about the contents of the video they had viewed. Previous studies had shown that the specificity induction improves performance on subsequent episodic tasks relative to both the math control induction and an impressions control induction requiring participants to report general thoughts and opinions of the viewed video; both control inductions require little specific episodic retrieval, and indistinguishable increases due to the specificity induction have been observed with either control induction as the baseline in young adults (Madore et al., 2015; Madore et al., 2014; Madore \& Schacter, 2016). We chose the math control induction for Experiment 1 because this control has not been used with older adults previously, and it is potentially a more neutral baseline than the impressions control (e.g., no retrieval orientation should be induced with the math control, whereas some sort of retrieval orientation could be induced with questions that have participants reflect back on the video in a general way). Participants spent approximately $6 \mathrm{~min}$ in both the specificity induction and the math control induction.

\section{Main tasks}

After receiving one of the two inductions, participants were introduced to the think aloud procedure, developed by Gilhooly et al. (2007), that would be used for the AUT, OAT, and imagination task. With this procedure, participants

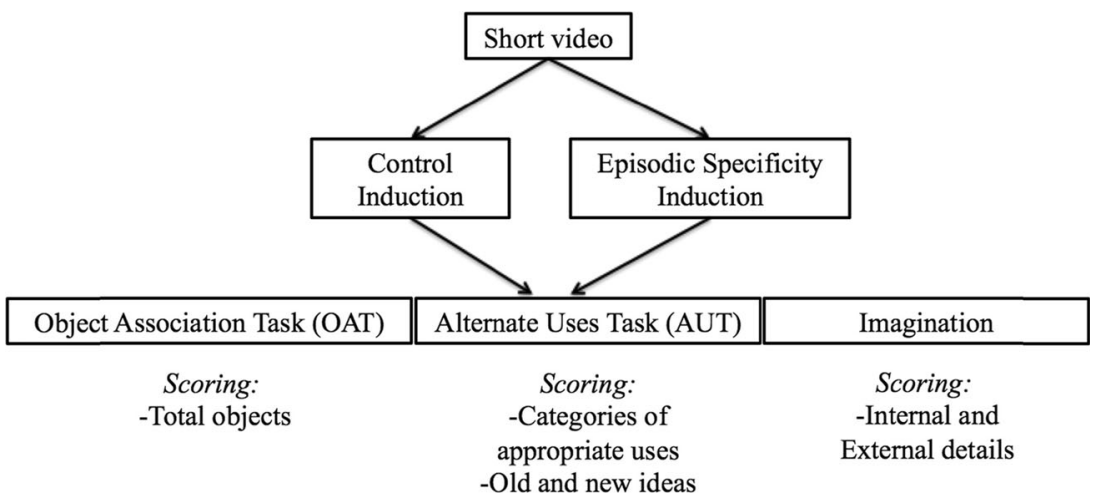

Fig. 1 Schema of the experimental design and main variables of interest in Experiment 1 
were told to talk out loud as they worked on each trial from beginning to end, to respond freely without planning what to say, and to act as if they were alone in the room speaking to themselves. Participants first practiced the think aloud procedure and then received more specific instructions for each of the three main tasks: AUT, OAT, and imagination. For the think aloud procedure and each main task, the respective instructions appeared on the computer screen in front of participants and were also read out loud by the experimenter, which served to equate expectations regarding the report criteria following both induction manipulations (see the supplemental materials for instruction sets).

During each trial of the main tasks, a different object cue was presented on the computer screen with a short task instruction reminder, and participants responded out loud accordingly. This screen appeared for the duration of each trial. For the AUT and OAT, participants also wrote out their answers as they verbalized their thoughts aloud (as in Gilhooly et al., 2007, with the AUT). Seventeen different object cues were used in each session (six per task for the AUT and OAT, and five per task for imagination). Participants completed one practice cue for each task, followed by the main experimental trials (i.e., five for the AUT and OAT, and four for imagination). The cues were common, everyday objects (e.g., newspaper, a bed sheet, eye glasses) appearing in the official test booklet for the AUT (Guilford, Christensen, Merrifield, \& Wilson, 1960) and in current studies of divergent thinking. The order of the tasks was blocked (e.g., OAT-AUT-imagination) to reduce cognitive load, and these blocked sequences were presented in a random order to participants; the order of the object cues was also randomized. Participants were audiorecorded as they worked on each trial, and the experimenter presented no inputs or questions during the main trials. A majority of the participants (70\%) were run by one of two experimenters blind to the study's hypotheses.

Because previous work has suggested that older adults sometimes perform differently on divergent-thinking tasks as a function of the time allowed per trial (e.g., Foos \& Boone, 2008; Leon et al., 2014), all participants completed each trial of the main tasks in a self-paced, untimed manner in which they simply pressed the space bar when they were ready to go on to the next trial.

Alternate uses task The AUT is a typical index of divergent thinking that requires combining different types of information in novel and flexible ways (e.g., Guilford, 1967; Guilford et al., 1960) and that involves episodic imagery (e.g., Addis et al., 2016; Madore et al., 2015). Participants were asked to report as many unusual and creative uses for each object cue as possible. They were told that each object cue had a common use and that they should come up with alternative ones (i.e., "for the object cue pencil case, focus on generating uses other than to hold pencils and pens"). We highlighted both creativity (i.e., "focus on generating creative and unusual uses") and fluency (i.e., "list as many other uses for the object cue as you can") in our instructions, on the basis of evidence that receiving one instruction over the other can impact divergentthinking performance (e.g., Nusbaum et al., 2014). At the end of the second session, participants viewed each use they had generated during the experiment and labeled each as an "old idea" (i.e., one from a previous memory or thought) or a "new idea" (i.e., one that had come to mind for the first time during the study) to examine the types of ideas that young and older adults generated as a function of induction (Benedek et al., 2014; Gilhooly et al., 2007; Silvia et al., 2015).

Object association task The OAT is a complement to the AUT that also requires combining different types of information in response to object cues, but with less reliance on divergent thinking and episodic imagery, and more emphasis on generating semantic information (based on Abraham et al., 2012; Madore et al., 2015). Participants were asked to report as many objects that were related to each object cue as possible. They were told that each object cue had a common use and that they should come up with typical semantic associates (i.e., "for the object cue pencil case, focus on generating objects like pencil, paper, desk, and so on").

Imagination As in previous studies of episodic future thinking (e.g., Addis et al., 2008; Madore et al., 2015), participants in this task were asked to generate one novel event that could happen on one day within the next few years in one place related to the object cue from a field (i.e., from one's own eye's) perspective (i.e., "if you imagine going on a vacation, think about one event that will happen to you on one day of the vacation rather than over the vacation as a whole"). Participants were instructed to imagine everything they could about the event they generated in as much detail as possible, including actions, people, and feelings. Given the robust induction-related and overall age-related effects on this task (see, e.g., Addis et al., 2008, 2016; Madore et al., 2014; Madore \& Schacter, 2014), we included it in the present study as a manipulation check on performance.

\section{Scoring}

Participants' responses were transcribed and scored by one of four independent raters blind to all experimental hypotheses and to which induction had been received. Before commencing the scoring of the experimental trials, the raters completed practice scoring and a subsequent interrater reliability assessment on the responses from young and older adults with high agreement (see below for the reliability statistics for each task).

AUT For the main analyses below, raters identified the number of categories of appropriate uses that participants 
generated (as in Madore et al., 2015, and as modified from Addis et al., 2016; Guilford, 1967; and Guilford et al., 1960). This dependent variable is the most stringent definition of a use, because it includes appropriate uses, or feasible and possible ones given the cue, that are clustered into distinct categories when possible. For example, a safety pin could be used as earrings or as a bracelet charm and would be clustered under one category of jewelry; using a safety pin as a mini gun or as a laser would fall under one category of weaponry but would not be scored because the uses were inappropriate. Raters were in high agreement on this variable in a sample of ten AUT responses (Cronbach's $\alpha=.92$ ). The total numbers of categories of appropriate uses were summed across the five experimental trials in each session to obtain an index of AUT performance.

Other standard dimensions of AUT performance were also evaluated, including total fluency, appropriate uses alone, categories of uses alone, an elaboration rating, and a creativity rating (based on Addis et al., 2016; Benedek et al., 2014; Guilford, 1967; Guilford et al., 1960; Madore et al., 2015). Total fluency is the total number of uses generated, excluding repetitions. Appropriate uses is the total number of uses that were feasible and possible given the cue. Categories of uses alone is the total number of unique categories that uses could be binned under for each cue (irrespective of appropriateness). Elaboration is a rating of how detailed each generated use was $(0=$ brief to $2=$ more detailed $)$. Creativity is a rating of how original/unusual and appropriate each generated use was ( $1=$ uncreative to $4=$ very creative $)$; the highest ratings of 3 and 4 were reserved for those uses that only a few people could presumably provide. High interrater reliability was obtained along the dimensions of appropriate uses (Cronbach's $\alpha=.99$ ), categories of uses (.92), elaboration (.87), and creativity (.94); total fluency was not scored by the raters, as it was simply a sum of the uses generated by each participant.

It should be noted that sometimes the AUT is scored for originality (with a certain number of points awarded for each response that is statistically infrequent in the sample, as described by Guilford, 1967). We did not score participants' responses for originality because potential biases are related to sample-specific scoring methods. For example, participants who generated similar additional uses with the specificity induction would not receive credit for doing so (i.e., these additional uses would become more statistically frequent in the sample), and thus any underlying effects of the manipulation would not be observed. We included the creativity rating (which is not sample-specific) to measure the originality of each response, instead.

To examine the numbers of old and new ideas generated on the AUT as a function of age group and induction, we simply summed the total numbers of responses that participants generated across the five experimental trials in each session, and split them into those that had been labeled "old" and those that had been labeled "new."

OAT For this task, raters identified object words that participants had generated, and excluded other words to ensure consistency with previous research on this topic (based on Abraham et al., 2012; Madore et al., 2015). For example, object associates for a safety pin such as diaper and shirt were counted, whereas a word like sewing was not counted. Raters were high in agreement on this variable in a sample of ten OAT responses (Cronbach's $\alpha=.99$ ). The total numbers of objects were summed across the five experimental trials in each session to obtain an index of OAT performance.

Imagination Raters coded the narratives that participants generated into internal details and external details (e.g., Addis et al., 2016; Levine et al., 2002; Madore et al., 2015). Internal details refer to episodic details contained in a central event generated by the participant (including people, setting, actions, feelings, objects, etc.). External details refer to semantic and off-topic details contained in the event narrative (including factual statements, commentary on the task itself, etc.). Raters were high in agreement for the internal details (Cronbach's $\alpha$ $=.95$ ) and external details (Cronbach's $\alpha=.88$ ) in a sample of 12 imagination responses. The total numbers of internal details and external details were summed for each of the four experimental trials separately in each session, and a trial-by-trial average for the session was computed.

\section{Results}

To address our hypotheses, we performed a series of mixedfactorial analyses of variance (ANOVAs) testing for main effects and interactions, with $\alpha=.05$. For imagination (see Table 1 ), the between-subjects factor Age (young vs. older) and the within-subjects factors Induction (control vs. specificity) and Detail Type (internal vs. external) were entered into the ANOVA model using the output variables of internal details and external details. Seventeen responses (4.62\% of the total) were excluded for not falling within the next few years. For the main divergent-thinking analyses (see Fig. 2), the between-subjects factor Age (young vs. older) and the within-subjects factors Induction (control vs. specificity) and Task (OAT vs. AUT) were entered into the ANOVA model using the output variables of total objects on the OAT and categories of appropriate uses on the AUT. No responses were excluded. For the old- versus new-ideas analyses (see Fig. 3), the between-subjects factor Age (young vs. older) and the within-subjects factors Induction (control vs. specificity) and Idea Type (old vs. new) were entered into the ANOVA model using the output variables of total AUT responses that were labeled either "old" or "new." One AUT response $(<1 \%$ of the total) was excluded for being unlabeled. We first present 
Table 1 Mean details generated by young and older adults in imagination as a function of induction

\begin{tabular}{|c|c|c|c|c|c|c|}
\hline & \multicolumn{3}{|l|}{ Young } & \multicolumn{3}{|l|}{ Older } \\
\hline & Control & Specificity & Collapsed & Control & Specificity & Collapsed \\
\hline Imagination internal details & $39.19(4.74)$ & $51.89(5.40)$ & $45.54(4.61)$ & $26.39(4.74)$ & $38.81(4.75)$ & $32.60(4.24)$ \\
\hline Imagination external details & $12.67(2.58)$ & $11.35(2.80)$ & $12.01(2.50)$ & $56.52(18.87)$ & $44.66(10.03)$ & $50.59(12.51)$ \\
\hline
\end{tabular}

The results are from Experiment 1. Numbers in parentheses represent $1 S E$

the age-related evidence for each task, followed by the induction-related evidence.

\section{Age-related evidence}

Imagination We examined whether age group impacted performance on the imagination task in terms of the internal details (i.e., episodic) and external details (e.g., semantic) generated, irrespective of induction. The main effect of age on the two detail types together was nonsignificant, $F(1,44)=2.63, p=$ $.112, \eta_{\mathrm{p}}{ }^{2}=.06$. Critically, we did observe a significant Age $\times$ Detail Type interaction, $F(1,44)=17.30, p<.001, \eta_{\mathrm{p}}{ }^{2}=0.28$. Post-hoc tests indicated that older adults generated significantly fewer internal details on the imagination task than did young adults, $t(44)=2.07, p=.045$, mean difference $=12.94,95 \% \mathrm{CI}$ $=[0.32,25.56], d=0.61$. Older adults also exhibited a significantly greater number of external details on the imagination task than did young adults, $t(44)=3.02, p=.004$, mean difference $=38.58,95 \% \mathrm{CI}=[12.87,64.29], d=0.89$.

Divergent thinking Given this evidence, we examined whether age group impacted performance on the AUT and OAT in terms of the categories of appropriate uses and total objects generated, irrespective of induction. The main effect of age on the two tasks together was nonsignificant, $F(1,44)=$ $0.62, p=.44, \eta_{\mathrm{p}}{ }^{2}=.01$. The Age $\times$ Task interaction was also nonsignificant, $F(1,44)=0.002, p=.97, \eta_{\mathrm{p}}^{2}=.001$. These findings indicate that young and older adults performed similarly on the AUT and OAT. The same pattern of nonsignificant results was obtained with the other dimensions of AUT use generation: total uses, appropriate uses, and categories of all uses. We also found no significant age-related differences for the ratings of elaboration or creativity.

Old versus new ideas We next examined the types of ideas generated by young and older adults on the AUT, irrespective of induction. Paralleling the main divergent-thinking analyses, we observed no main effect of age on the total number of ideas (i.e., old and new) generated on the AUT, $F(1,44)=0.08, p=$ $.78, \eta_{\mathrm{p}}{ }^{2}=.002$. The Age $\times$ Idea Type interaction was also nonsignificant, $F(1,44)=0.07, p=.79, \eta_{\mathrm{p}}^{2}=.002$. These results indicate that young and older adults did not differ in their production of old and new ideas on the AUT.

\section{Induction-related evidence}

Imagination To assess whether the specificity induction operated as expected, and to assess for the first time whether older adults exhibit an induction effect using the neutral math control baseline, we examined whether the manipulation impacted the numbers of internal details (i.e., episodic) and external details (e.g., semantic) generated by young and older adults on the imagination task. We found no main effect of induction on the two detail types together, $F(1,44)=0.40, p=$
A

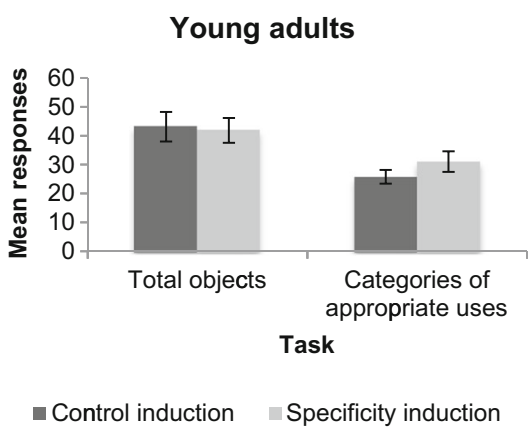

B

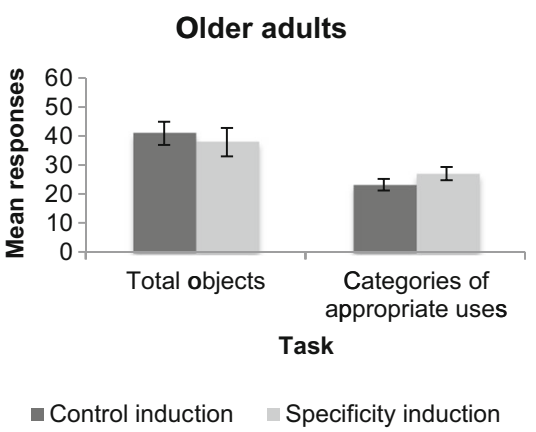

Fig. 2 Experiment 1: Mean responses generated for total objects on the object association task and for categories of appropriate uses on the alternate uses task by young adults (A) and older adults (B) as a function of induction. Error bars represent $1 S E$ 
A

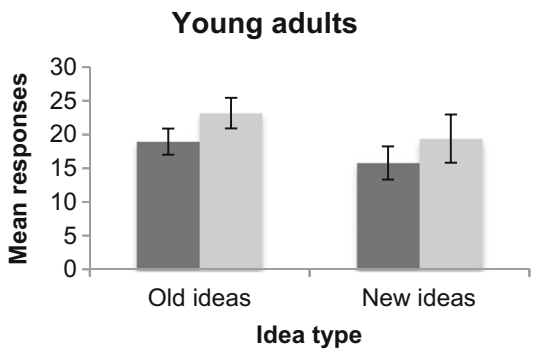

- Control induction Specificity induction
B

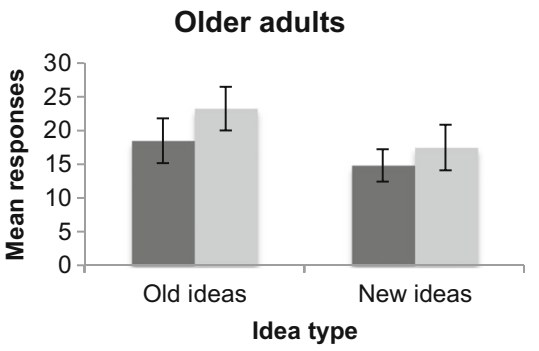

- Control induction Specificity induction

Fig. 3 Experiment 1: Mean responses labeled "old ideas" and "new ideas" for total uses on the alternate uses task by young adults (A) and older adults (B) as a function of induction. Error bars represent $1 S E$

$.53, \eta_{\mathrm{p}}{ }^{2}=.01$. Critically, however, there was a significant Induction $\times$ Detail Type interaction, $F(1,44)=4.94, p=$ $.031, \eta_{\mathrm{p}}{ }^{2}=.10$, and the Induction $\times$ Age and Induction $\times$ Detail Type $\times$ Age interactions were nonsignificant $\left(F_{\mathrm{s}} \leq\right.$ $0.36, p s \geq .55$ ). Post-hoc tests indicated that both young and older adult participants generated significantly more internal details on the imagination task after receiving the specificity induction rather than the control, $t(45)=4.21, p<.001$, mean difference $=12.56,95 \% \mathrm{CI}=[6.55,18.58], d=0.62$. No significant differences in external details were exhibited as a function of induction, $t(45)=0.78, p=.44$, mean difference $=$ $6.59,95 \% \mathrm{CI}=[-23.66,10.48], d=0.11$.

Divergent thinking Given that the specificity induction operated as expected on the imagination task, we examined whether the manipulation impacted the number of categories of appropriate uses generated on the AUT and the number of total objects generated on the OAT in young and older adults. No main effect of induction was apparent on the two tasks together, $F(1$, $44)=0.48, p=.49, \eta_{\mathrm{p}}{ }^{2}=.01$. Critically, we did observe a significant Induction $\times$ Task interaction, $F(1,44)=5.32, p=$ $.026, \eta_{\mathrm{p}}{ }^{2}=.11$, and once again the Induction $\times$ Age and Induction $\times$ Task $\times$ Age interactions were nonsignificant $(F \mathrm{~s}$ $\leq 0.22, p s \geq .64$ ). Post-hoc tests indicated that both young and older adults generated significantly more categories of appropriate uses on the AUT following the specificity induction as compared with the control, $t(45)=3.19, p=.003$, mean difference $=4.54,95 \% \mathrm{CI}=[1.68,7.41], d=0.47$. There was no significant difference between young and older adults in total objects generated on the OAT following the specificity induction as compared with the control, $t(45)=0.76, p=.45$, mean difference $=2.15,95 \% \mathrm{CI}=[-7.83,3.53], d=0.11$.

We also ran a series of mixed-factorial ANOVAs with the between-subjects factor Age Group (young vs. older) and the within-subjects factors Induction (control vs. specificity) and Task (OAT vs. AUT), examining total uses, appropriate uses, and categories of uses against total objects, and found the same pattern of results. Critically, in each analysis the Induction $\times$ Task interaction was significant $\left(F_{\mathrm{S}} \geq 5.12\right.$; for total fluency, $p=.01, \eta_{\mathrm{p}}{ }^{2}=.14$; for appropriate uses, $p=.009$, $\eta_{\mathrm{p}}{ }^{2}=.14$; and for categories of uses, $p=.029, \eta_{\mathrm{p}}{ }^{2}=.10$ ) but the Induction $\times$ Age and Induction $\times$ Task $\times$ Age interactions were nonsignificant $\left(F_{\mathrm{s}} \leq 0.20, p \mathrm{~s} \geq .66\right)$. Both young and older adults provided significantly more total uses $\left(M_{\text {control }}\right.$ $\left.=34.04, S E=2.58 ; M_{\mathrm{spec}}=41.57, S E=3.62\right)$, appropriate uses $\left(M_{\text {control }}=33.74, S E=2.55 ; M_{\text {spec }}=41.43, S E=3.60\right)$, and categories of uses $\left(M_{\text {control }}=24.76, S E=1.57 ; M_{\text {spec }}=\right.$ 29.17, $S E=2.10$ ) following the specificity induction than after the control, smallest $t(45)=3.05, p=.004$, mean difference $=$ $4.41,95 \% \mathrm{CI}=[1.50,7.33], d=0.48$.

Participants' uses were also rated as being both similarly elaborate $\left(M_{\text {control }}=0.62, S E=0.08 ; M_{\mathrm{spec}}=0.64, S E=0.06\right)$, $F(1,44)=0.12, p=.73, \eta_{\mathrm{p}}{ }^{2}=.003$, and similarly creative $\left(M_{\text {control }}=2.30, S E=0.07 ; M_{\text {spec }}=2.25, S E=0.06\right), F(1,44)$ $=0.94, p=.34, \eta_{\mathrm{p}}{ }^{2}=.02$, following both inductions; we examined the total uses variable for elaboration and creativity (the same nonsignificant pattern was observed with the other generative dimensions). These findings indicate that the qualities of the responses generated via both inductions in young and older adults were similar.

Old versus new ideas Given the selective effects of the specificity induction on generative AUT performance, we next examined whether the manipulation affected the production of total ideas labeled as "old" and "new" in young and older adults. The main effect of induction on total ideas on the AUT (i.e., old + new) was significant, $F(1,44)=10.19, p=.003$, $\eta_{\mathrm{p}}{ }^{2}=.19$. Critically, the Induction $\times$ Idea Type interaction here was not significant, $F(1,44)=0.35, p=.56, \eta_{\mathrm{p}}{ }^{2}=.01$, and the Induction $\times$ Age and Induction $\times$ Idea Type $\times$ Age interactions were also not significant $\left(F_{\mathrm{s}} \leq 0.11, p \mathrm{~s} \geq .75\right)$. There was a trend for more old ideas than new ideas to be generated on the AUT overall (i.e., a main effect of idea type), $F(1,44)=3.25$, $p=.078, \eta_{\mathrm{p}}{ }^{2}=.07$, but this did not reach full significance. These findings suggest that the specificity induction significantly increased the numbers of both old and new ideas generated on the AUT in young and older adults, as compared with the control induction. 


\section{Discussion}

In Experiment 1, our manipulation check confirmed that the specificity induction was operating as expected: Both young and older adults generated significantly more internal details (i.e., episodic) but not external details (e.g., semantic) on imagination following the specificity induction than in the control condition. This result replicates previous findings with young adults (e.g., Madore et al., 2014), and provides novel evidence that older adults show similar induction effects as compared with a neutral math control baseline (previous studies showing specificity induction effects on imagination in older adults had used an impressions control baseline that could conceivably have reduced performance below baseline levels; for discussion, see Madore \& Schacter, 2014). Critically, receiving the specificity induction also led to significantly and similarly enhanced generative performance on the AUT in young and older adults as compared with a control induction, while having no significant effect on the OAT. This pattern of results replicates previous work with young adults (Madore et al., 2015) and extends it for the first time to older adults, thereby suggesting that episodic memory processes are involved in divergent thinking for both age groups. In line with earlier studies, we also found typical overall age-related differences on the imagination task (see Schacter, Gaesser, \& Addis, 2013, for a review). However, we did not find overall age-related differences on the AUT or OAT, a point to which we will return in the General Discussion.

Although we have argued that the finding of a significant specificity induction effect on generative AUT performance in older adults implicates episodic memory in divergent-thinking performance, the finding that older adults benefited as much from the induction as did younger adults, despite abundant evidence of impaired episodic retrieval in the elderly, could suggest that the induction has its impact on a process other than episodic retrieval, such as some aspect of visual imagery that is elicited by the induction, affects AUT but not OAT performance, and is preserved in older adults. Though such a possibility cannot be ruled out definitively, we think that the most parsimonious interpretation of our results is that the induction serves to activate episodic retrieval processes in older adults that they would normally not activate on their own. Numerous previous investigators have emphasized that older adults exhibit deficits in self-initiated, controlled retrieval processes (cf. Craik, 1983; Jennings \& Jacoby, 1993; Koutstaal, 2003; Luo \& Craik, 2009) and that the specificity induction may help counter such deficits.

\section{Experiment 2}

To further examine the role of episodic retrieval in divergent thinking, in Experiment 2 we assessed whether the specificity induction would affect performance on a second divergentthinking measure in young adults, the consequences task (Guilford, 1967; Torrance, 1962). This task requires generating the consequences of improbable and novel scenarios that do not exist in real life (e.g., living on without death). We chose this task because it is another frequently used index of divergent thinking, and also because it should rely less on memories from the personal past than does the AUT; participants should not have many, if any, memories involving the cues used on the consequences task, given that the cues involve scenarios that are not part of everyday life. Although the results from Experiment 1 and previous work (Madore et al., 2015) suggest that the specificity induction boosts both old and new idea production on divergent thinking, as we noted earlier, in some previous experiments the induction effect seems to have been driven more strongly by the production of old ideas from memory than by new ideas from imagination, even though the Induction $\times$ Idea Type interactions are nonsignificant. It is known that participants who complete the AUT sometimes draw on particular episodic memories (Gilhooly et al., 2007; Runco \& Acar, 2010), so it is important to assess induction effects on a divergent-thinking task that is less likely to elicit such memories. We chose the consequences task because it should elicit a higher proportion of new ideas than the AUT, and thus allow for a clearer assessment of whether the specificity induction reliably affects new-idea output on divergent thinking. As in our previous induction work (Madore et al., 2015) and in Experiment 1 of the present study on the AUT, we hypothesized that the specificity induction would lead to enhanced performance on the consequences task as compared with a control, in terms of the total numbers of consequences generated, appropriate consequences, categories of consequences, and categories of appropriate consequences; we did not expect differences in ratings of elaboration or creativity as a function of induction. We also hypothesized that new-idea production would be boosted by the manipulation. This hypothesis fits with the constructive episodic simulation hypothesis (Schacter \& Addis, 2007), which emphasizes that elements of prior experiences may be retrieved and recombined to create novel episodic representations.

\section{Method}

\section{Participants}

Thirty-two young adults (age $=18-28$ years, $M=20.69, S D=$ 2.47; 21 female, 11 male) were run in Experiment 2 with the same recruitment and data collection parameters as in Experiment 1. Their educational levels were also similar to those observed in Experiment $1(M=15.00$ years, $S D=$ 1.24). Prior to beginning the study, we decided on a sample size of 32 participants, because this had been an adequate number for observing medium-sized induction effects in a 
previous study in which young adults received both a specificity induction and a control induction in a single session (i.e., Madore \& Schacter, 2016). No participants were excluded from the final analyses.

\section{Overview, materials, and procedure}

Participants came to the lab for a single-session study in which they underwent two segments. In each segment, participants (a) watched one of the two short videos used in Experiment 1, (b) received either the episodic specificity induction or an impressions control induction after watching the video, and (c) then completed the consequences task of divergent thinking. Participants completed a brief, 5-min math filler after finishing the first segment and before beginning the second segment of the study. As in Experiment 1, the video-induction order was counterbalanced across participants, who were randomly assigned to receive the specificity induction in the first segment and the impressions control in the second segment, or the impressions control in the first segment and the specificity induction in the second segment. This experimental design was the same as that used in a previous study in which participants had switched between inductions in one session (i.e., Madore \& Schacter, 2016). The session took on average 1.5 to $2 \mathrm{~h}$ to complete. Figure 4 illustrates the experimental sequence and variables of interest.

We replaced the math control induction with the impressions control induction because the latter is potentially a more stringent baseline. In the impressions control induction (see the supplemental materials for the full script), participants first verbalized their global opinions, thoughts, and impressions of the video they had viewed, and then responded to a number of questions from a question bank focused on general information related to the setting, people, and actions of the video, as well as its other elements. For example, participants were asked what adjectives they would use to describe the video, what

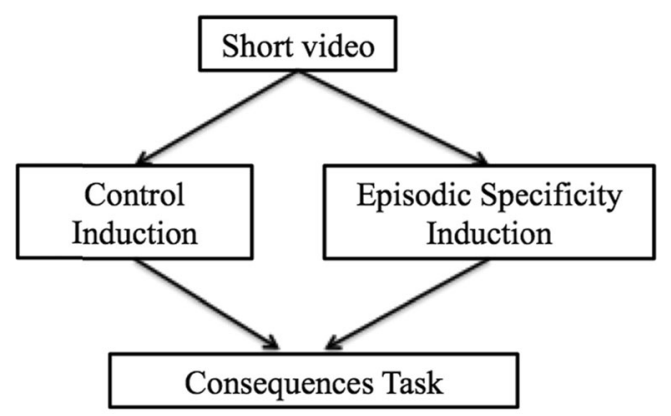

$$
\begin{gathered}
\text { Scoring: } \\
\text {-Categories of } \\
\text { appropriate consequences } \\
\text {-Old and new ideas }
\end{gathered}
$$

Fig. 4 Schema of the experimental design and main variables of interest in Experiment 2 equipment they thought had been used to make it, and when they thought it was made. No mental-imagery probes were used. As we mentioned, previous work found indistinguishable increases in performance on subsequent episodic tasks when the specificity induction was compared with the impressions control or the math control (Madore et al., 2015; Madore et al., 2014; Madore \& Schacter, 2016). We chose the impressions control for Experiment 2 because it is well matched with the specificity induction in terms of requiring participants to reflect and report on information related to the viewed video; the main difference between the two inductions is the degree to which specific episodic information is retrieved. Participants spent approximately $6 \mathrm{~min}$ in each induction.

After receiving either the specificity induction or the impressions control induction, participants completed the consequences task (Guilford, 1967; Torrance, 1962). The consequences task is a typical index of divergent thinking that requires participants to generate the consequences of several improbable, novel scenarios (e.g., flying without mechanical aids). In Experiment 2, participants viewed five different improbable, novel scenarios in each segment (as listed by Torrance, 1962; for examples of studies using this task, see Foos \& Boone, 2008, and Ononye, BlinnPike, \& Smith, 1993) and were asked to generate as many consequences of each scenario as possible. Participants were also instructed to be as unusual and creative as possible, to match the instructions from Experiment 1 for the AUT, and also given previous research (e.g., Nusbaum et al., 2014) suggesting that stressing creativity or fluency can impact divergent thinking (see the supplemental materials for the instructions and cue sets). Participants viewed each scenario on the computer screen in front of them with a short task reminder, and typed out their answers in text boxes that appeared on the screen; when the trial ended after $5 \mathrm{~min}$, the screen automatically moved to the next cue. Two blocks of five cues were created (i.e., one block per segment), and the block order was counterbalanced across participants and inductions. A majority of the participants ( $72 \%$ ) were run by one of two experimenters blind to the study's hypotheses, and the experimenter presented no probes or inputs during the trials.

After completing the two segments (i.e., one induction and one round of consequences, a filler task, and the second induction and second round of consequences), participants were shown each consequence they had generated for each scenario in the experiment and were asked to label each as an "old idea" or "new idea" (as in Exp. 1 and Gilhooly et al., 2007). Participants then rated how new and unusual they found each scenario itself to be, completed category and fluency measures, and responded to the same four questionnaires as in Experiment 1 (see the supplemental materials).

\section{Scoring}

Participants' responses on the consequences task were later scored by one of two independent raters blind to all 
experimental hypotheses and to which induction had been received. As in Experiment 1, we focused on categories of appropriate consequences for the main analysis, because this is the most stringent definition of a response (based on Guilford, 1967, and Torrance, 1962; see Alpaugh et al., 1982, for related scoring). Responses were rated as appropriate, or feasible and possible given the cue, and were clustered into distinct categories when possible. For example, for the cue flying without mechanical aids, the consequences of new air traffic laws and new passport laws would be binned under one category of rules. Before viewing the experimental trials, the raters completed practice scoring and underwent an interrater reliability assessment on ten responses from the consequences task with young adults, with high agreement (Cronbach's $\alpha=.92$ ). The total numbers of categories of appropriate consequences across the five trials of each segment were summed for the main index of divergent-thinking performance.

As in Experiment 1, other dimensions of creativity were evaluated, including total fluency, appropriate consequences alone, categories of consequences alone, an elaboration rating, and a creativity rating. High interrater reliabilities were obtained for appropriate consequences (Cronbach's $\alpha=.99$ ), categories of consequences (.92), elaboration (.85), and creativity (.93); total fluency was simply a sum of all consequences and was not scored by the raters. It should be noted that sometimes the consequences task is scored for obvious consequences (i.e., those consequences produced by over $5 \%$ of the sample) and remote consequences (i.e., those consequences produced by under $5 \%$ of the sample), as outlined by Guilford (1967) and Torrance (1962). Like originality in Experiment 1, we did not score for this dimension in Experiment 2 because of potential biases in samplespecific scoring methods with statistical frequencies; we instead relied on the creativity rating (which is not sample-specific) to measure originality.

For the old- and new-idea analyses, we simply summed the total numbers of consequences labeled as "old" and "new" across the five experimental trials in each segment.

\section{Results}

To assess whether the specificity induction impacted performance on the consequences task, we ran a series of repeated measures ANOVAs with the within-subjects factor Induction (control vs. specificity) and $\alpha=.05$. For the main divergentthinking analysis (see Fig. 5), the output variable was categories of appropriate consequences. For the old- versus new-ideas analysis (see Fig. 6), we included Idea Type (old vs. new) as another within-subjects factor in the ANOVA model using the output variables total old consequences and total new consequences. No responses were excluded from either analysis.

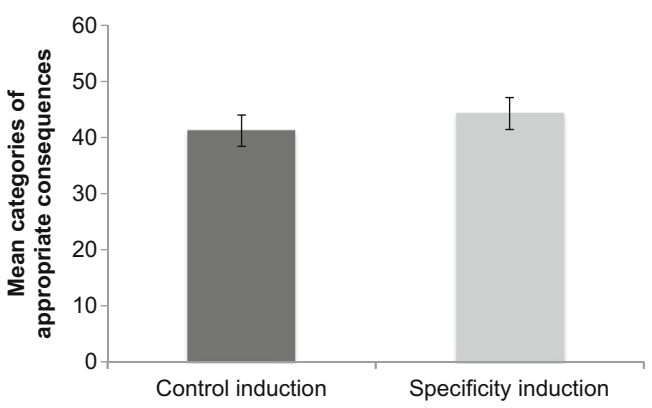

Fig. 5 Experiment 2: Mean responses generated for categories of appropriate consequences on the consequences task by young adults, as a function of induction. Error bars represent $1 S E$

\section{Divergent thinking}

We first examined whether receiving the specificity induction affected subsequent performance on the consequences task in terms of the categories of appropriate consequences. We found a significant main effect of induction, $F(1,31)=$ $23.86, p<.001, \eta_{\mathrm{p}}{ }^{2}=.44$, mean difference $=3.06,95 \% \mathrm{CI}$ $=[1.78,4.34], d=0.87$, indicating that participants generated a significantly greater number of categories of appropriate consequences following the specificity induction than following the control.

The same pattern of results was obtained using the other generative dimensions on the consequences task, with a series of repeated measures ANOVAS for total consequences, appropriate consequences, and categories of consequences. Critically, in each analysis a main effect of induction emerged ( $F \mathrm{~S} \geq 11.42$; for total fluency, $p=.002, \eta_{\mathrm{p}}{ }^{2}=.27$; for appropriate uses, $p=.002, \eta_{\mathrm{p}}{ }^{2}=.27$; and for categories of uses, $p<$ $\left..001, \eta_{\mathrm{p}}{ }^{2}=.46\right)$, indicating that significantly more total consequences $\left(M_{\text {control }}=63.94, S E=3.92 ; M_{\text {spec }}=71.28, S E=\right.$ 4.18), appropriate consequences $\left(M_{\text {control }}=63.84, S E=3.94\right.$; $\left.M_{\text {spec }}=70.91, S E=4.11\right)$, and categories of consequences $\left(M_{\text {control }}=41.19, S E=2.79 ; M_{\text {spec }}=44.34, S E=2.85\right)$ were produced following the specificity induction than after the control, smallest $t(31)=3.38, p=.002$, mean difference $=$ 7.06, $95 \% \mathrm{CI}=[2.80,11.32], d=0.60$.

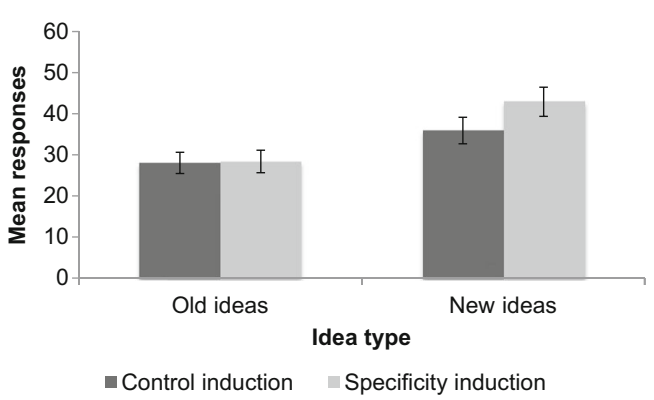

Fig. 6 Experiment 2: Mean responses labeled "old ideas" and "new ideas" for total consequences on the consequences task by young adults, as a function of induction. Error bars represent $1 S E$ 
As in Experiment 1, participants' consequences were rated as being similarly elaborate $\left(M_{\text {control }}=0.77, S E=0.08 ; M_{\text {spec }}\right.$ $=0.86, S E=.10), F(1,31)=3.56, p=.069, \eta_{\mathrm{p}}{ }^{2}=.10$, and similarly creative $\left(M_{\text {control }}=2.56, S E=0.06 ; M_{\text {spec }}=2.54, S E\right.$ $=0.06), F(1,31)=0.33, p=.57, \eta_{\mathrm{p}}{ }^{2}=.01$, following both inductions, suggesting that the qualities of responses were similar across inductions. We examined the total consequences variable for both elaboration and creativity (the same nonsignificant results were obtained with the other generative dimensions).

Old versus new ideas Given this result, we next examined the impact of the specificity induction on the types of ideas generated on the consequences task, in terms of old versus new. There was a significant main effect of induction, $F(1,31)=$ $11.61, p=.002, \eta_{\mathrm{p}}{ }^{2}=.27$, indicating that participants generated significantly more total consequences (i.e., old + new) following the specificity induction than in the control condition. The main effect of idea type was also significant, $F(1,31)$ $=6.92, p=.013, \eta_{\mathrm{p}}{ }^{2}=.18$, indicating that more new than old ideas were generated on the consequences task. Specifically, $57 \%$ of the ideas on the consequences task were characterized as "new," as compared with $42 \%$ on the AUT in Experiment 1. Critically, we observed a significant Induction $\times$ Idea Type interaction on performance, $F(1,31)=4.39, p=.044, \eta_{\mathrm{p}}{ }^{2}=$ .12. Post-hoc tests indicated that a significantly greater number of new ideas was generated following the specificity induction than following the control, $t(31)=3.26, p=.003$, mean difference $=7.00,95 \% \mathrm{CI}=[2.63,11.37], d=0.58$. No significant difference was apparent in the number of old ideas generated as a function of induction, $t(31)=0.21, p=$ .84 , mean difference $=0.34,95 \% \mathrm{CI}=[-3.05,3.74], d=0.04$.

\section{General discussion}

The two experiments reported here extend our knowledge of episodic specificity induction effects on divergent thinking in three main ways. First, Experiment 1 showed for the first time that previously observed specificity induction effects on the AUT in young adults are observed to a similar degree in older adults. Second, Experiment 2 showed for the first time that the specificity induction boosts performance on another test of divergent thinking, the consequences task. Third, both experiments indicate that the effects of the specificity induction are not restricted to old ideas that reflect retrieval of particular episodic memories, but can be reliably observed for new ideas that have not been previously experienced. Indeed, in Experiment 2 the beneficial effects of the specificity induction on the consequences task occurred only for new ideas. The induction-related effects on aging in Experiment 1 and on a second creativity index in Experiment 2 strengthen the idea that episodic retrieval is involved in divergent thinking (in terms of the number of responses generated), even for novel ideas beyond those contained in past experiences.

The finding that performance on the AUT in older adults benefits as much from an episodic specificity induction as the performance of young adults extends previous observations of comparable specificity induction effects on young and older adults in memory, imagination, and means-end problemsolving tasks (Madore et al., 2014; Madore \& Schacter, 2014) that are thought to make use of episodic memory processes. The main difference between the previous results and those in the present study is that older adults exhibited reduced levels of performance relative to young adults after both specificity and control inductions on memory, imagination, and problem-solving tasks, whereas here they exhibited intact performance on the AUT in both conditions. Although caution should be used in interpreting null results, as we noted earlier, the previous literature concerning generative AUT performance in older adults is mixed. A number of factors could contribute to this mixed pattern. From a methodological standpoint, allowing young and older adults to complete divergentthinking tasks in an untimed format, as in the present study, versus a timed format has been found to attenuate age-related differences on such tasks (e.g., Foos \& Boone, 2008; Leon et al., 2014).

Divergent thinking also elicits different cognitive processes than do imagination or means-end problem-solving tasks on which older adults exhibit impaired performance (cf. Addis et al., 2008; Gaesser et al., 2011; Sheldon, McAndrews, \& Moscovitch, 2011). The latter tasks emphasize retrieving and recombining episodic information to construct novel scenarios (Madore et al., 2014; Madore \& Schacter, 2014), whereas divergent thinking requires building multiple mini-scenarios (with different uses or consequences), which may more naturally involve recombining episodic and semantic information (Gilhooly et al., 2007). Because older adults typically have intact crystallized intelligence and semantic memory (i.e., general knowledge), they may perform as well as or better than young adults on cognitive tasks like divergent thinking, which rely on or emphasize semantic knowledge for completion (see Alpaugh et al., 1982, and Leon et al., 2014, for related discussions). In other words, older adults may compensate for deficits in episodic processes by relying more on intact semantic processes to complete divergent-thinking tasks, a strategy that could minimize the observance of agerelated effects.

This sort of interpretation fits well with the data of the present study, in which age-related differences were found on the imagination task (with fewer internal/episodic details and more external/semantic details produced by older than by young adults), but not on the AUT or the OAT. Nonetheless, developing a well-validated and standardized index or dependent measure of divergent thinking that can reliably distinguish between episodic and semantic contributions to task responses could 
help explain the contrasting age-related findings on tasks like the AUT. Studies that further probe and distinguish the strategies and types of information used to complete divergent thinking (Gilhooly et al., 2007; Runco \& Acar, 2010) should help clarify this issue. It should also be noted that strategies and processes beyond retrieving and recombining elements of episodic and semantic memory have been examined in previous studies of divergent thinking, including but not limited to working memory, inhibition, attention, and vocabulary (e.g., Gilhooly et al., 2007; Leon et al., 2014; McCrae et al., 1987; Reese et al., 2001; Zabelina et al., 2015). Young and older adults may differ in the extents to which they recruit these processes on creativity tasks, in particular, which could contribute to the mixed pattern of age-related results, and which should be studied further in future work.

Although divergent thinking recruits nonepisodic processes, our present findings and previous observations (Madore et al., 2015) that an episodic specificity induction boosts generative divergent thinking on the AUT and on the consequences task highlight that episodic processes also contribute to divergent thinking. However, the consequences task involves responding to improbable, novel scenarios, and thus should not draw heavily on access to particular episodic memories for individual experiences, as was confirmed by our finding that participants provide primarily "new" ideas on this task. Indeed, we found that only new ideas - but not old ideas were significantly boosted in Experiment 2 following the specificity induction, as compared with the control.

Some interpretive caution is called for when considering the data concerning old and new ideas. As in related research on the sources of creative ideas (Benedek et al., 2014; Gilhooly et al., 2007; Silvia et al., 2015), we adopted a retrospective method of labeling uses as "old" or "new," rather than asking participants to label ideas as "old" or "new" during task performance, to avoid biases in the sorts of ideas that participants thought they should generate during the experiment itself. However, this retrospective method does have its limitations, because source confusions could arise from retrospective judgments, and the induction manipulation itself is thought to impact how information is retrieved, perhaps influencing what is labeled an "old" or a "new" idea. An important task for future research will be to develop improved methods for distinguishing between old and new ideas on divergent-thinking tasks.

How can an induction that targets episodic retrieval affect new-idea production? In line with previous induction effects on imagination and means-end problem solving (Madore et al., 2014; Madore \& Schacter, 2014), the manipulation could be targeting the retrieval of elements of prior experiences that are recombined to construct novel episodic representations that have not been experienced before. As we suggested earlier, this idea is in line with a central claim of the constructive episodic simulation hypothesis (Schacter \& Addis, 2007), that elements of past experiences may be flexibly recombined to form novel episodic representations. In a related vein, the induction may be targeting a scene construction (Hassabis \& Maguire, 2007) or event construction (Romero \& Moscovitch, 2012) process that is a critical component of episodic retrieval and that is common to memory, imagination, means-end problem solving, and divergent thinking (see Schacter \& Madore, in press, for further theoretical elaboration). By scene or event construction, we mean assembling and maintaining a mental scene or event by filling it in with details related to people, actions, and objects; this process is related to episodic retrieval because elements of prior experiences may be utilized to fill in the details of a mental scene or event. Because the induction targets the specific retrieval and construction of a mental representation involving particular elements of a previously experienced scene or event, including people, objects, and actions, it should affect performance on subsequent cognitive tasks that may nominally be facilitated by this scene or event construction process, including but not limited to divergent thinking. As applied to divergent thinking, episodic retrieval could affect the recollection of alternate uses from previous experiences (i.e., old ideas) and the generation of novel ideas that have not been experienced before but rely on the recombination of elements of prior experiences (i.e., new ideas). This interpretation fits well with the present pattern of results: In Experiment 1, the induction boosted old- and new-idea production on the AUT, and in Experiment 2, the induction boosted new-idea production on the consequences task.

A few features of the consequences task may explain why we did not find effects of the induction on old-idea production in Experiment 2. From a methodological standpoint, only a relatively small number of old ideas were generated on this task. The induction effect was somewhat stronger for old-idea production in Experiment 1 (with overall old- and new-idea production at $58 \%$ and $42 \%$, respectively), whereas the effect was stronger for new-idea production in Experiment 2 (with overall old- and new-idea production at $43 \%$ and $57 \%$, respectively). In addition, the specificity induction may not have affected old-idea production on the consequences task, because the old ideas by definition may have been primarily semantic in nature or based on general knowledge for this task (e.g., factual and abstracted knowledge drawn from movies or novels). The prompts involved improbable, novel scenarios that do not exist in real life, and so the degree to which participants could access particular episodes from the past to complete the prompts was very limited. The restriction of access to particular episodes is less of an issue for new-idea production on the consequences task, because responses to the prompts could be generated by retrieving, integrating, and recombining the elements of different experiences to form novel episodic representations. Future studies should continue to investigate the parameters under which old and new ideas are generated on divergent-thinking tasks.

The behavioral evidence from the present experiments and related work also complements recent neural evidence that 
similar core networks of brain regions are involved in episodic memory, imagination, and divergent thinking (Benoit \& Schacter, 2015; Beaty, Benedek, Silvia, \& Schacter, 2016). Numerous studies have directly compared brain activity when people remembered past experiences and imagined future experiences (for a review, see Schacter et al., 2012), but no published study has directly compared remembering and imagining with divergent thinking. Future work should examine all three cognitive tasks in a single fMRI experiment to assess the similarities and differences in the processes recruited for such tasks. The results from our behavioral experiments suggest that the core processes involved in episodic memory, including the retrieval of prior elements of experiences to construct representations of scenes or events, can contribute to divergent thinking.

Author note This research was supported by National Institute on Aging Grant No. AG08441 to D.L.S. K.P.M. is supported by the Sackler Scholar Programme in Psychobiology. We thank Haley Dodds, Shenghan (Zoe) Lu, Bradley Perkins, Samantha F. Schoenberger, Kristina Tummino, and Emelia Vigil for assistance with various aspects of the study.

\section{References}

Abraham, A., Pieritz, K., Thybusch, K., Rutter, B., Kroger, S., Schweckendiek, J.,...Hermann, C. (2012). Creativity and the brain: Uncovering the neural signature of conceptual expansion. Neuropsychologia, 50, 1906-1917. doi:10.1016/j. neuropsychologia.2012.04.015

Addis, D. R., Pan, L., Musicaro, R., \& Schacter, D. L. (2016). Divergent thinking and constructing episodic simulations. Memory, 24, 89-97. doi:10.1080/09658211.2014.985591

Addis, D. R., Wong, A. T., \& Schacter, D. L. (2008). Age-related changes in the episodic simulation of future events. Psychological Science, 19, 33-41. doi:10.1111/j.1467-9280.2008.02043.x

Alpaugh, P., \& Birren, J. E. (1977). Variables affecting creative contributions across the adult life-span. Human Development, 20, 240-248. doi:10.1159/000271559

Alpaugh, P. K., Parham, I. A., Cole, K. D., \& Birren, J. E. (1982). Creativity in adulthood and old age: An exploratory study. Educational Gerontology, 8, 101-116. doi:10.1080/ 0380127820080202

Beaty, R. E., Benedek, M., Silvia, P. J., \& Schacter, D. L. (2016). Creative cognition and brain network dynamics. Trends in Cognitive Sciences, 20, 87-95. doi:10.1016/j.tics.2015.10.004

Benedek, M., Jauk, E., Fink, A., Koschutnig, K., Reishofer, G., Ebner, F., \& Neubauer, A. C. (2014). To create or to recall? Neural mechanisms underlying the generation of creative new ideas. NeuroImage, 88, 125-133. doi:10.1016/j.neuroimage.2013.11.021

Benoit, R. G., \& Schacter, D. L. (2015). Specifying the core network supporting episodic simulation and episodic memory by activation likelihood estimation. Neuropsychologia, 75, 450-457. doi:10. 1016/j.neuropsychologia.2015.06.034

Craik, F. I. M. (1983). On the transfer of information from temporary to permanent memory. Philosophical Transactions of the Royal Society of London B, 302, 341-359. doi:10.1098/rstb.1983.0059
Duff, M. C., Kurczek, J., Rubin, R., Cohen, N. J., \& Tranel, D. (2013). Hippocampal amnesia disrupts creative thinking. Hippocampus, 23, 1143-1149. doi:10.1002/hipo.22208

Faul, F., Erdfelder, E., Lang, A., \& Buchner, A. (2007). G*Power 3: A flexible statistical power program for the social, behavioral, and biomedical sciences. Behavior Research Methods, 39, 175-191. doi:10.3758/BF03193146

Fisher, R. P., \& Geiselman, R. E. (1992). Memory-enhancing techniques for investigative interviewing: The cognitive interview. Springfield, IL: Charles C. Thomas.

Foos, P. W., \& Boone, D. (2008). Adult age differences in divergent thinking: It's just a matter of time. Educational Gerontology, 34, 587-594. doi:10.1080/03601270801949393

Gaesser, B., Sacchetti, D. C., Addis, D. R., \& Schacter, D. L. (2011). Characterizing age-related changes in remembering the past and imagining the future. Psychology and Aging, 26, 80-84. doi:10. 1037/a0021054

Gilhooly, K. J., Fioratou, E., Anthony, S. H., \& Wynn, V. (2007). Divergent thinking: Strategies and executive involvement in generating novel uses for familiar objects. British Journal of Psychology, 98, 611-625. doi:10.1348/096317907X173421

Guilford, J. P. (1967). The nature of human intelligence. New York: McGraw Hill.

Guilford, J. P., Christensen, P. R., Merrifield, P. R., \& Wilson, R. C. (1960). Alternate uses manual. Menlo Park, CA: Mind Garden, Inc.

Hassabis, D., \& Maguire, E. A. (2007). Deconstructing episodic memory with construction. Trends in Cognitive Sciences, 11, 299-306. doi: 10.1016/j.tics.2007.05.001

Jennings, J. M., \& Jacoby, L. L. (1993). Automatic versus intentional uses of memory: Aging, attention, and control. Psychology and Aging, 8, 283-293. doi:10.1037/0882-7974.8.2.283

Jing, H. G., Madore, K. P., \& Schacter, D. L. (2016). Worrying about the future: An episodic specificity induction impacts problem solving, reappraisal, and well-being. Journal of Experimental Psychology: General, 145, 402-418. doi:10.1037/xge0000142

Koutstaal, W. (2003). Older adults encode - but do not always useperceptual details: Intentional versus unintentional effects of detail on memory judgments. Psychological Science, 14, 189-193. doi:10. 1111/1467-9280.01441

Leon, S. A., Altmann, L. J. P., Abrams, L., Gonzalez Rothi, L. J., \& Heilman, K. M. (2014). Divergent task performance in older adults: Declarative memory or creative potential? Creativity Research Journal, 26, 21-29. doi:10.1080/10400419.2014.873657

Levine, B., Svoboda, E., Hay, J. F., Winocur, G., \& Moscovitch, M. (2002). Aging and autobiographical memory: Dissociating episodic from semantic retrieval. Psychology and Aging, 17, 677-689. doi: 10.1037/0882-7974.17.4.677

Luo, L., \& Craik, F. I. M. (2009). Age differences in recollection: Specificity effects at retrieval. Journal of Memory and Language, 60, 421-436. doi:10.1016/j.jml.2009.01.005

Madore, K. P., Addis, D. R., \& Schacter, D. L. (2015). Creativity and memory: Effects of an episodic-specificity induction on divergent thinking. Psychological Science, 26, 1461-1468. doi:10.1177/ 0956797615591863

Madore, K. P., Gaesser, B., \& Schacter, D. L. (2014). Constructive episodic simulation: Dissociable effects of a specificity induction on remembering, imagining, and describing in young and older adults. Journal of Experimental Psychology: Learning, Memory, and Cognition, 40, 609-622. doi:10.1037/a0034885

Madore, K. P., \& Schacter, D. L. (2014). An episodic specificity induction enhances means-end problem solving in young and older adults. Psychology and Aging, 29, 913-924. doi:10.1037/a0038209

Madore, K. P., \& Schacter, D. L. (2016). Remembering the past and imagining the future: Selective effects of an episodic specificity induction on detail generation. The Quarterly Journal of 
Experimental Psychology, 69, 285-298. doi:10.1080/17470218. 2014.999097

McCrae, R. R. (1987). Creativity, divergent thinking, and openness to experience. Journal of Personality and Social Psychology, 52, 1258-1265. doi:10.1037/0022-3514.52.6.1258

McCrae, R. R., Arenberg, D., \& Costa, P. T. (1987). Declines in divergent thinking with age: Cross-sectional, longitudinal, and crosssequential analyses. Psychology and Aging, 2, 130-137. doi:10. 1037/0882-7974.2.2.130

Mednick, S. A. (1962). The associative basis of the creative process. Psychological Review, 69, 220-232. doi:10.1037/h0048850

Memon, A., Meissner, C. A., \& Fraser, J. (2010). The cognitive interview: A meta-analytic review and study space analysis of the past 25 years. Psychology, Public Policy, and Law, 16, 340-372. doi:10. 1037/a0020518

Nusbaum, E. C., Silvia, P. J., \& Beaty, R. E. (2014). Ready, set, create: What instructing people to "be creative" reveals about the meaning and mechanisms of divergent thinking. Psychology of Aesthetics, Creativity, and the Arts, 8, 423-432. doi:10.1037/a0036549

Ononye, G. C., Blinn-Pike, L. M., \& Smith, D. E. (1993). Creativity and future time perspective: Exploring fantasy and realistic measures. Creativity Research Journal, 6, 449-456. doi:10.1080/ 10400419309534499

Palmiero, M. (2015). The effects of age on divergent thinking and creative objects production: A cross-sectional study. High Ability Studies, 26, 93-104. doi:10.1080/13598139.2015.1029117

Palmiero, M., Di Giacomo, D., \& Passafiume, D. (2014). Divergent thinking and age-related changes. Creativity Research Journal, 26, 456-640. doi:10.1080/10400419.2014.961786

Reese, H. W., Lee, L., Cohen, S. H., \& Puckett, J. M. (2001). Effects of intellectual variables, age, and gender on divergent thinking in adulthood. International Journal of Behavioral Development, 25, 491500. doi:10.1080/01650250042000483

Romero, K., \& Moscovitch, M. (2012). Episodic memory and event construction in aging and amnesia. Journal of Memory and Language, 67, 270-284. doi:10.1016/j.jml.2012.05.002

Roskos-Ewoldsen, B., Black, S. A., \& McCown, S. M. (2008). Agerelated changes in creative thinking. The Journal of Creative Behavior, 42, 33-59. doi:10.1002/j.2162-6057.2008.tb01079.x

Runco, M. A., \& Acar, S. A. (2010). Do tests of divergent thinking have an experiential bias? Psychology of Aesthetics, Creativity, and the Arts, 4, 144-148. doi:10.1037/a0018969
Runco, M. A., Okuda, S. M., \& Thurston, B. J. (2014). The psychometric properties of four systems for scoring divergent thinking tests. Journal of Psychoeducational Assessment, 5, 149-156. doi:10. 1177/073428298700500206

Ruth, J., \& Birren, J. E. (1985). Creativity in adulthood and old age: Relations to intelligence, sex and mode of testing. International Journal of Behavioral Development, 8, 99-109. doi:10.1177/ 016502548500800107

Schacter, D. L., \& Addis, D. R. (2007). The cognitive neuroscience of constructive memory: Remembering the past and imagining the future. Philosophical Transactions of the Royal Society of London B, 362, 773-786. doi:10.1098/rstb.2007.2087

Schacter, D. L., Addis, D. R., Hassabis, D., Martin, V. C., Spreng, R. N., \& Szpunar, K. K. (2012). The future of memory: Remembering, imagining, and the brain. Neuron, 76, 677-694. doi:10.1016/j. neuron.2012.11.001

Schacter, D. L., Gaesser, B., \& Addis, D. R. (2013). Remembering the past and imagining the future in the elderly. Gerontology, 59, 143151. doi: $10.1159 / 000342198$

Schacter D. L., \& Madore K. P. (in press). Remembering the past and imagining the future: Identifying and enhancing the contribution of episodic memory. Memory Studies.

Sheldon, S., McAndrews, M. P., \& Moscovitch, M. (2011). Episodic memory processes mediated by the medial temporal lobes contribute to open-ended problem solving. Neuropsychologia, 49, 2439-2447. doi:10.1016/j.neuropsychologia.2011.04.021

Silvia, P. J., Nusbaum, E. C., \& Beaty, R. E. (2015). Old or new? Evaluating the old/new scoring method for divergent thinking tasks. The Journal of Creative Behavior. doi:10.1002/jocb.101. Advance online publication.

Torrance, P. E. (1962). Guiding creative talent. Englewood Cliffs, NJ: Prentice-Hall.

Tulving, E. (1983). Elements of episodic memory. Oxford: Clarendon Press.

Tulving, E. (2002). Episodic memory: From mind to brain. Annual Review of Psychology, 53, 1-25. doi:10.1146/annurev.psych.53. 100901.135114

Zabelina, D. L., O’Leary, D., Pornpattananangkul, N., Nusslock, R., \& Beeman, M. (2015). Creativity and sensory gating indexed by the P50: Selective versus leaky sensory gating in divergent thinkers and creative achievers. Neuropsychologia, 69, 77-84. doi:10.1016/j. neuropsychologia.2015.01.034 